\title{
Mechanical stimulation orchestrates the osteogenic differentiation of human bone marrow stromal cells by regulating HDAC1
}

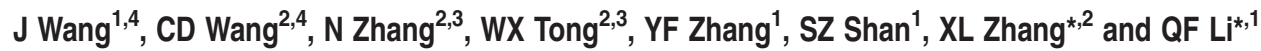

Mechanical stimulation and histone deacetylases (HDACs) have essential roles in regulating the osteogenic differentiation of bone marrow stromal cells (BMSCs) and bone formation. However, little is known regarding what regulates HDAC expression and therefore the osteogenic differentiation of BMSCs during osteogenesis. In this study, we investigated whether mechanical loading regulates HDAC expression directly and examined the role of HDACs in mechanical loading-triggered osteogenic differentiation and bone formation. We first studied the microarrays of samples from patients with osteoporosis and found that the NOTCH pathway and skeletal development gene sets were downregulated in the BMSCs of patients with osteoporosis. Then we demonstrated that mechanical stimuli can regulate osteogenesis and bone formation both in vivo and in vitro. NOTCH signaling was upregulated during cyclic mechanical stretch (CMS)-induced osteogenic differentiation, whereas HDAC1 protein expression was downregulated. The perturbation of HDAC1 expression also had a significant effect on matrix mineralization and JAG1-mediated Notch signaling, suggesting that HDAC1 acts as an endogenous attenuator of Notch signaling in the mechanotransduction of BMSCs. Chromatin immunoprecipitation (ChIP) assay results suggest that HDAC1 modulates the CMSinduced histone $\mathrm{H} 3$ acetylation level at the JAG1 promoter. More importantly, we found an inhibitory role of Hdac1 in regulating bone formation in response to hindlimb unloading in mice, and pretreatment with an HDAC1 inhibitor partly rescued the osteoporosis caused by mechanical unloading. Our results demonstrate, for the first time, that mechanical stimulation orchestrates genes expression involved in the osteogenic differentiation of BMSCs via the direct regulation of HDAC1, and the therapeutic inhibition of HDAC1 may be an efficient strategy for enhancing bone formation under mechanical stimulation.

Cell Death and Disease (2016) 7, e2221; doi:10.1038/cddis.2016.112; published online 12 May 2016

Osteoporosis is a debilitating bone disease that can occur when bone marrow stromal cells (BMSCs) fail to produce a sufficient number of osteoblasts to counteract bone resorption by osteoclasts. The fate of BMSCs is determined based on the integration of chemical (including bone morphogenetic proteins (BMPs), Wnt, MAPKs, and Notch signaling), ${ }^{1}$ spatial and physical signals. ${ }^{2-5}$ Mechanical stretching has previously been reported to be an important regulator of diverse biological and pathological processes. ${ }^{6}$ It has been convincingly demonstrated that tensile force induces osteogenic differentiation and that compression force induces chondrogenic differentiation. ${ }^{7,8}$ An analysis of the effects of cyclic mechanical stimulation on the osteogenic differentiation of human MSCs shows that $10 \%$ cyclic tensile strain $(0.5 \mathrm{~Hz})$ enhances the activity of alkaline phosphatase (ALP) and the rate calcium deposition. ${ }^{9}$ Mechanical stimulation induces osteoblast marker gene expression and the secretion of hormones and growth factors, which affects the differentiation potential of BMSCs and modulates bone remodeling and homeostasis. Clinically, decreases in mechanical loading may result in significant bone loss in weight-bearing (WB) bones and, subsequently, a rapid progression of osteoporosis. ${ }^{10,11}$ However, a greater understanding of the role of mechanical loading in regulating the differentiation of human BMSCs is required.

In recent years, multiple microRNAs (miRNAs) ${ }^{12-14}$ and histone modification enzymes ${ }^{15}$ have been found to regulate osteogenic marker gene expression and osteogenesis

\footnotetext{
${ }^{1}$ Department of Plastic and Reconstructive Surgery, Shanghai Ninth People's Hospital, Shanghai Jiao Tong University School of Medicine, Shanghai 200011, China; ${ }^{2}$ The Key Laboratory of Stem Cell Biology, Institute of Health Sciences, Shanghai Institutes for Biological Sciences (SIBS), Chinese Academy of Sciences (CAS) and Shanghai Jiao Tong University School of Medicine, Shanghai 200023, China and ${ }^{3}$ Department of Orthopaedics and Traumatology, Prince of Wales Hospital, The Chinese University of Hong Kong, Shatin, New Territories 999077, Hong Kong

*Corresponding author: X Zhang, The Key Laboratory of Stem Cell Biology, Institute of Health Sciences, Shanghai Institutes for Biological Sciences (SIBS), Chinese Academy of Sciences (CAS) and Shanghai Jiao Tong University School of Medicine, Shanghai 200023, China. Tel: +86 2123271699 ext 5615; Fax: +86 21 63089567; or Q Li, Department of Plastic and Reconstructive Surgery, Shanghai Ninth People's Hospital, Shanghai Jiao Tong University School of Medicine, Shanghai 200011 , China. Tel: +86 2123271699 ext 5615; Fax: +86 2163089567.

E-mail: xlzhang@ sibs.ac.cn or dr.liqingfeng@shsmu.edu.cn

${ }^{4}$ These authors contributed equally to this work.

Abbreviations: BMSC, bone marrow stromal cell; CMS, cyclic mechanical stretch; ChIP, chromatin immunoprecipitation; ALP, alkaline phosphatase; gRT-PCR, quantitative real-time PCR; BMP, bone morphogenetic protein; Osx, osterix; PPAR, peroxisome proliferator-activated receptor; Col, collagen; Ocn, osteocalcin; aP2, fatty acid-binding protein-4; Glut4, glucose transporter type 4; PBS, phosphate-buffered saline; GSEA, Gene Set Enrichment Analysis; HU, hindlimb unloading; WB, weight bearing; H3, histone H3; Ac, acetylation; Runx2, runt-related transcription factor 2; JAG1, jagged 1; Hes1, hes family bHLH transcription factor 1; Hey1, hes-related family bHLH transcription factor with YRPW motif 1; HDAC, histone deacetylase

Received 18.11.15; revised 21.3.16; accepted 24.3.16; Edited by D Aberdam
} 
in vitro. ${ }^{16,17}$ The family of histone deacetylase (HDAC) enzymes comprises at least 18 genes classified into four groups, including class I (HDAC1, HDAC2, HDAC3, and HDAC8) and class II (HDAC4, HDAC5, HDAC6, HDAC7, HDAC9, and HDAC10). ${ }^{18}$ The inhibition of HDAC1 activity typically leads to the activation of transcription. During osteogenesis, total HDAC enzymatic activity is decreased, with a significant reduction in HDAC1 expression. Consistent with this finding, the recruitment of HDAC1 to the promoters of osteoblast marker genes, including osterix (Osx) and osteocalcin (Ocn), is downregulated, whereas histone $\mathrm{H} 3$ and $\mathrm{H} 4$ are hyperacetylated at those promoters during osteogenic differentiation. ${ }^{19}$ Previous studies have also shown that the suppression of HDAC activity with HDAC inhibitors accelerates osteogenesis. ${ }^{20}$ However, little is known regarding what regulates HDAC expression during osteogenesis. In addition, the functional roles of HDACs in the mechanotransduction of BMSCs have not been well characterized and are, therefore, particularly interesting to study.

BMSCs are simultaneously exposed to chemical and mechanical cues. In this study, we found that HDAC1 was negatively correlated with osteogenic differentiation and bone formation in the BMSCs of patients with osteoporosis, whereas jagged 1 (JAG1)-mediated NOTCH signaling was upregulated. Specifically, mechanical loading directly induced a downregulation of HDAC1 expression, which was involved in the promotion of osteogenic differentiation and bone formation through the targeting of JAG1, a master inducer of osteogenic differentiation. ${ }^{21-23}$ Our findings also demonstrate that the therapeutic inhibition of HDAC1 may partly rescue osteoporosis caused by mechanical unloading. This study may provide a novel mechanism and potential therapeutic target for enhancing bone formation under mechanical stimulation.

\section{Results}

Skeletal development and Notch signaling pathways were impaired in BMSCs from patient with osteoporosis. In this study, we compared the transcriptomes of BMSCs from four patients (aged 79-94 years) suffering from primary osteoporosis with the transcriptomes of BMSCs from an agematched control group (BMSCs-old; donor age, 79-89 years). Genome-wide gene expression patterns were examined using microarray hybridizations (The microarrays were downloaded from the GEO database, GEO accession number GSE35958). Using the GSEA method, we found that the cell cycle checkpoint, Notch signaling pathway, and skeletal development gene sets were significantly enriched in the BMSCs from the control group (the NES scores and FDR values for the gene sets were 2.054, 0.008; 1.748, 0.004; and $2.003,0.06$, respectively) compared with the results from the primary osteoporosis group (Figure 1a). By generating a heat map for gene products that were differentially expressed by at least twofold in the BMSCs from the osteoporosis group relative to their expression in the BMSCs from the age-matched control group, we were able to highlight the differences between BMSCs from the osteoporosis and control groups (Figure 1b). Osteoporotic cells exhibited a distinct gene expression profile independent of cellular aging, in which the components from the NOTCH signaling pathway, JAG1, JAG2, NOTCH1, and NOTCH2, were downregulated, and the expression of their target gene, HES1, was also decreased. As skeletal development or bone formation activity, the markers of osteogenic differentiation of BMSCs, such as RUNX2, COL1a2, and BMPR1B, were decreased in the osteoporosis group. The expression of genes coding for enhancers of osteoblast differentiation and matrix mineralization (SPP1, ALPL, EFNB2, COL1A1, and ANKH) was also reduced (data shown by Benisch et al. ${ }^{24}$ ). These results show that osteogenic differentiation and the related $\mathrm{NOTCH}$ signaling were impaired in the BMSCs of patients with osteoporosis.

To investigate the effects of mechanical loading that primarily regulate bone development in vivo, we adopted the HU mouse model, which has been widely used to simulate weightlessness and to study various aspects of musculoskeletal loading. For comparison with the effects of WB in the control group mice (WB, 6-month-old age-matched adult mice), HU mice were elevated by their tail for 28 days. Microcomputed tomography (microCT) showed that the bone volume of the tibial plateau in $\mathrm{HU}$ mice was significantly lower than that in WB mice (Figures 1c and d).

Mechanical stimulation orchestrates NOTCH signaling and HDACs in the osteogenic differentiation of BMSCs. We further analyzed the expression of Jag1-Notch signaling and Hdac genes in the BMSCs of the HU group. By isolating BMSCs from the WB and HU groups, we found that, without mechanical loading, the mRNA and protein expression of Notch1, Notch2, Jag1, Jag2, and their target genes Hes1 and Hey1 were all downregulated (Figures $2 a$ and b). Corresponding to the reduced Notch signaling, ALP staining and in vitro mineralization were also decreased in the $\mathrm{HU}$ group (Figure 2c). These results show that osteogenic differentiation was diminished in the absence of mechanical stimulation. Although the markers of osteogenic differentiation (Alp, Ocn, collagen type I, alpha 1 (Col1a1), and Osx) were downregulated, the markers of adipogenic differentiation (PPARy (peroxisome proliferator-activated receptor $\gamma$ ), aP2, Glut4) were significantly upregulated (Figures $2 d$ and e) in the HU group. The mRNA and protein expression of class I Hdacs (Hdac1, Hdac2, Hdac3) was enhanced, but the expression of class II Hdacs (Hdac5) was not (Figures $2 f$ and g). However, no evident differences in proliferation were found between the two groups after mechanical loading/ unloading for 3 weeks in our study (Figure $2 \mathrm{~h}$ ).

To investigate how mechanical loading regulates osteogenic differentiation in vitro, we developed the CMS-induced osteogenic differentiation model of human BMSCs. After loading for 3 weeks, qRT-PCR analysis showed that the expression of the osteogenic marker genes ALP, OCN, and COL1a1 was increased in the CMS group compared with their expression in the non-loading control cells (Ctrl group) (Figure 3a), which was confirmed by the results of the western blotting analysis (Figure $3 b$ ). Consistent with the above changes, $10 \%$ CMS treatment also enhanced ALP staining (Figure $3 \mathrm{c}$ ) and in vitro mineralization, as assessed by Alizarin red staining of mineralized deposits in the extracellular matrix 


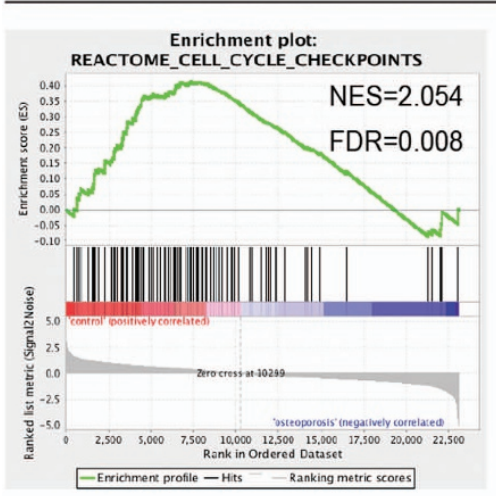

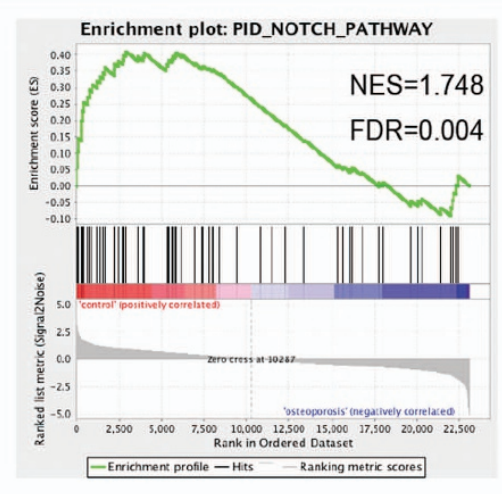

C

WB
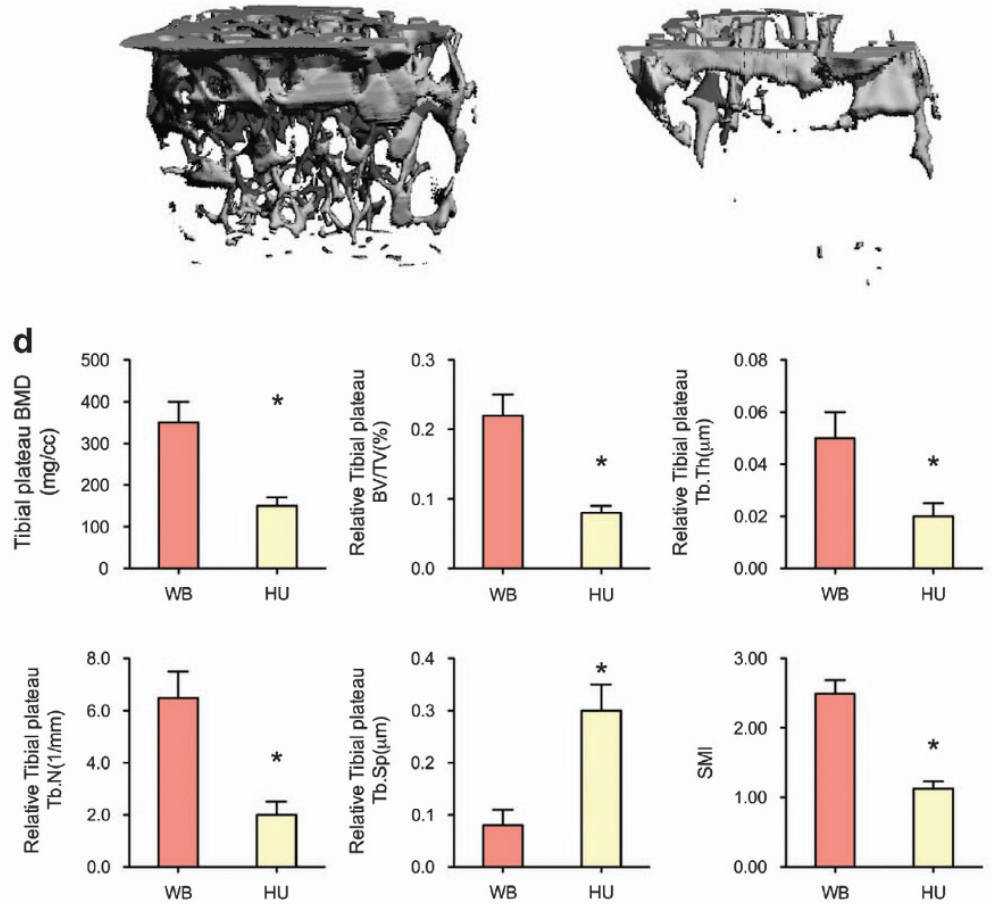

Figure 1 Mechanical loading positively regulates bone formation in vivo. (a) GSEA of expression profiles of BMSCs from normal (control) and osteoporosis patients. Enrichment curves computed by GSEA are shown in green (FDR-corrected $P<0.05$ ). GSEA for cell cycle checkpoints, Notch signaling pathway, and skeletal development gene sets demonstrated significant enrichment in control human BMSCs as compared with BMSCs from osteoporosis patients. (b) The heat map is ordered by degree of differential expression of Notch signaling pathway and skeletal development genes between BMSCs from normal (control) and osteoporosis patients. (c) Representative microCT reconstructive images of tibial plateau of WB and HU mice. $n=6$. (d) Three-dimensional microstructural parameters of tibial plateau of WB and HU mice. Data were mean \pm S.D., ${ }^{*} P<0.01$. All $P$-values are based on Student's t-test

(Figure 3d). These results show that CMS treatment enhances the osteogenic differentiation of BMSCs. Using immunofluorescence, we found an increased expression of Notch intracellular domain (NICD) and nucleus-accumulated RUNX2 after CMS for 3 days, which indicates the activation of NOTCH signaling and osteogenic differentiation (Figure $3 \mathrm{e}$ ). Moreover, CMS also promoted the mRNA and protein expression of the ligand for the NOTCH receptor JAG1 and the downstream genes of the NOTCH signaling pathway HES1 and HEY1
(Figures $3 \mathrm{f}-\mathrm{h}$ ). The protein and mRNA levels of HDAC1 were markedly decreased after CMS for 2 weeks, but no significant difference was observed after CMS for 3 weeks (Figure 3i). To confirm the role of JAG1 in osteogenesis, we inhibited JAG1 using a specific siRNA in the CMS-induced osteogenic differentiation model in human BMSCs, and we found that the CMS-induced mRNA and protein expression of JAG1 and the osteogenic markers COL1a1 and OCN were blocked by JAG1 siRNA (Figures $4 a-c$ ). The CMS-promoted osteogenic 
a

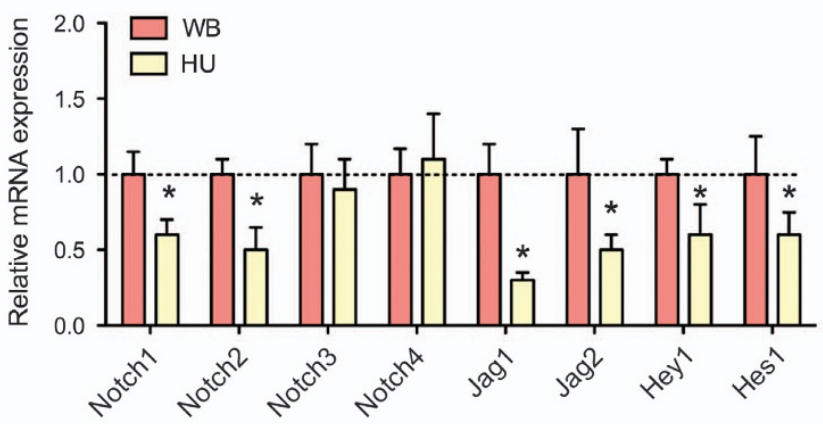

C

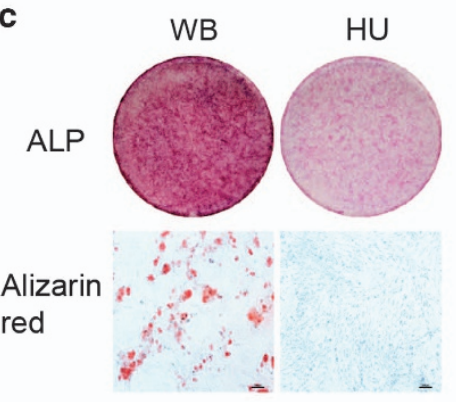

f

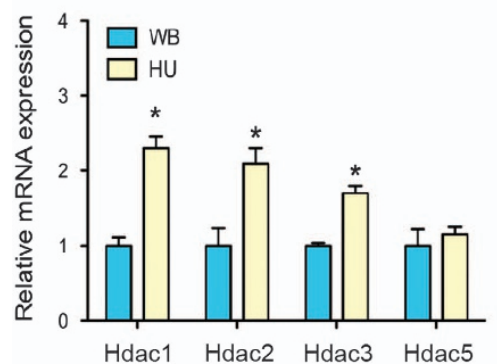

d

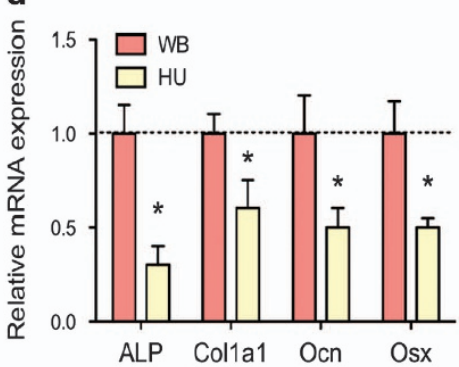

g

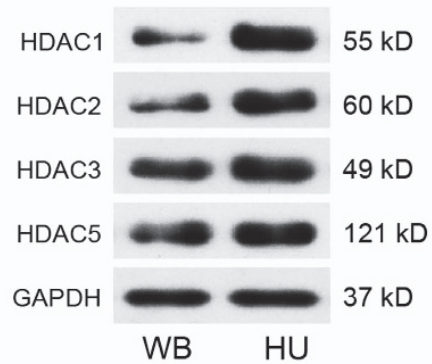

b

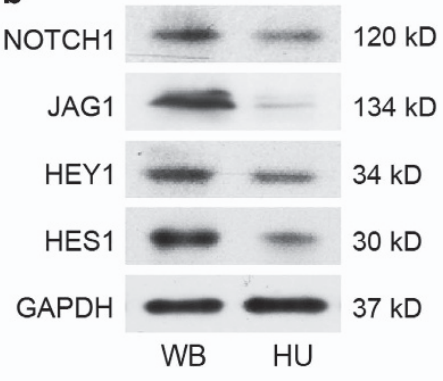

e

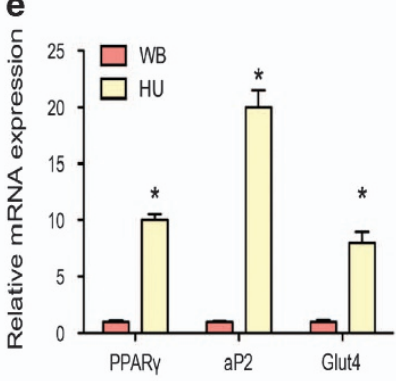

h

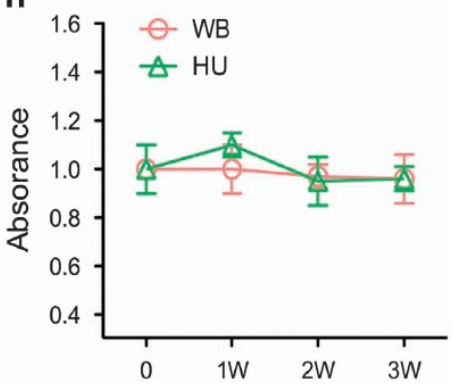

Figure 2 Notch signaling pathways and osteogenic differentiation were impaired in BMSCs of HU mice. (a) qRT-PCR analysis of Notch1-4, Jag1, Jag2, Hes1, and Hey1 mRNA levels in BMSCs of WB and HU mice. (b) Western blotting analysis of NOTCH1, JAG1, HES1, and HEY1 protein levels in BMSCs of WB and HU mice. (c) Representative images of ALP staining and Alizarin red staining of BMSCs from WB and HU mice. Scale bar, $100 \mu \mathrm{m}$. (d) qRT-PCR analysis of osteogenic differentiation markers Alp, Col1a1, Ocn, and Osx in BMSCs of WB and HU mice. (e) qRT-PCR analysis of adipogenic differentiation markers PPAR $\gamma$, aP2, and Glut4 in BMSCs of WB and HU mice. (f) qRT-PCR analysis and (g) western blotting analysis of HDACs in BMSCs of WB and HU mice. GAPDH was used as an internal control. (h) Cell viability was examined by MTT assay between BMSCs of WB and HU mice. All results are representative of at least three independent experiments. All the staining data were confirmed by three repeated tests. Data were mean \pm S.D., ${ }^{\star} P<0.01$. All $P$-values are based on Student's $t$-test

differentiation of BMSCs was also reduced by JAG1 inhibition, as shown by the in vitro mineralization results (Figure $4 d$ ). All of our results demonstrate that mechanical stimulation promotes the osteogenic differentiation of BMSCs by activating JAG1-mediated pro-osteogenic Notch signaling and reducing the expression of HDAC1.

HDAC1 modulates JAG1-Notch signaling during CMSinduced osteogenic differentiation. Next we examined whether there was a connection between the increased JAG1 expression and the decreased HDAC1 expression during CMS-induced osteogenesis, as acetylation of histones has been implicated in the activation of transcription. Previous studies have shown that suppression of HDAC activity using HDAC inhibitors accelerates osteogenesis. ${ }^{20}$ To clarify the role of HDAC1 in regulating CMS-induced osteogenesis, we treated human BMSCs with an HDAC1 inhibitor or caused them to overexpress HDAC1. First, we inhibited HDAC1 using a specific siRNA in the CMS-induced osteogenic differentiation model. Without effecting JAG1 expression (Figures $4 \mathrm{e}$ and $\mathrm{f}$ ), we found that the CMSinduced expression of the osteogenic markers ALP, COL1a1, and OCN were enhanced by HDAC1 siRNA treatment, which was further supported by the ALP staining results (Figure $4 \mathrm{~g}$ ). Furthermore, we investigated the influence of HDAC1 on the expression of the JAG1-NOTCH signaling pathway and, therefore, on osteogenesis. We found that the CMS-activated mRNA and protein expression of JAG1, HES1, and HEY1 were facilitated by HDAC1 inhibition (Figures $4 \mathrm{~h}$ and i). Interestingly, as shown by the in vitro mineralization assay, CMS-activated osteogenesis was enhanced by HDAC1 inhibition. However, this enhancement by HDAC1 inhibition was significantly blocked by an inhibitor of $\mathrm{NOTCH}$ signaling transduction (10 nM RO4929097, an inhibitor of $\gamma$ secretase) 


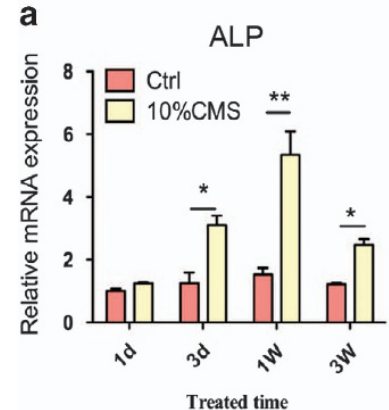

c
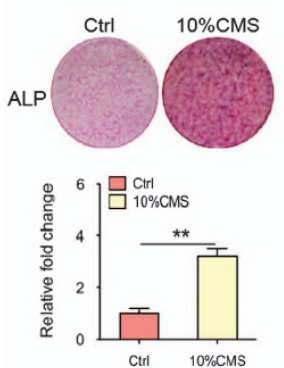

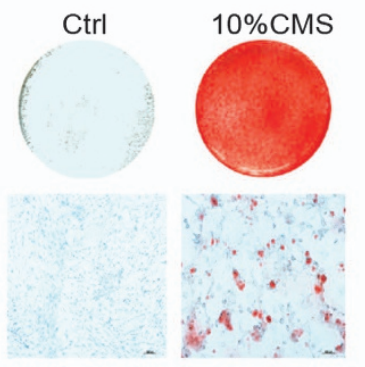

COL1a1

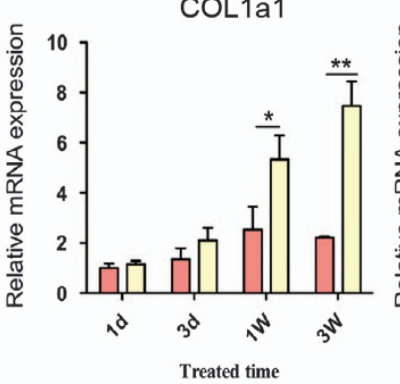

Treated time d

f

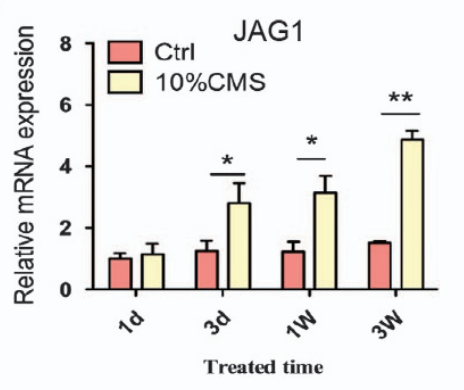

g.

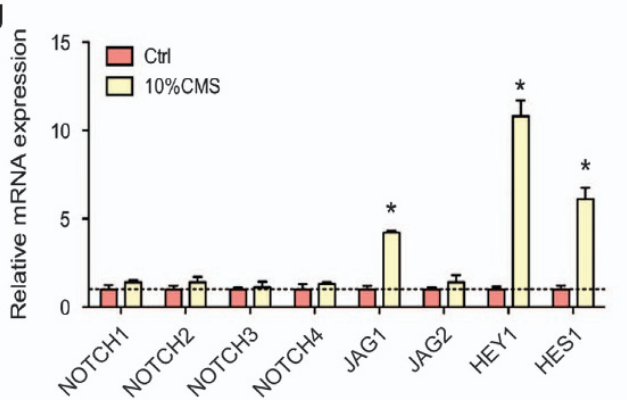

b

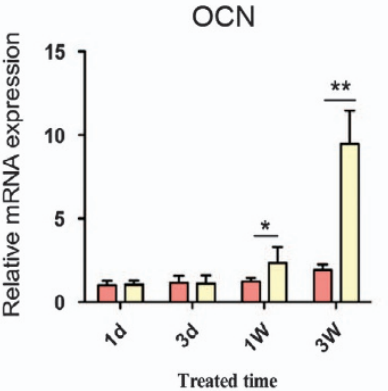

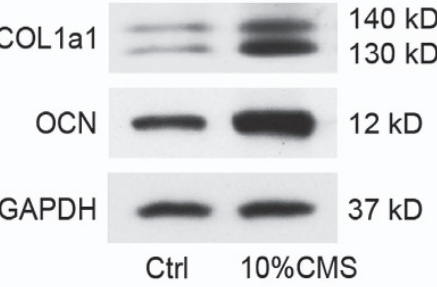

$\mathrm{OCN}$

$\mathrm{kD}$

e

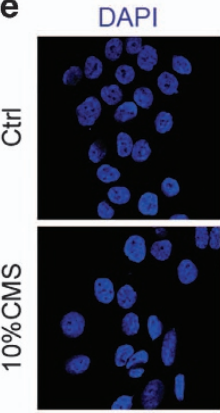

RUNX2

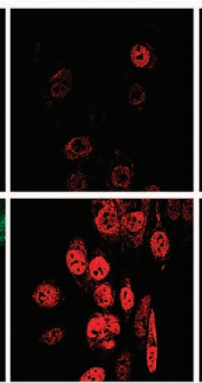

Merge

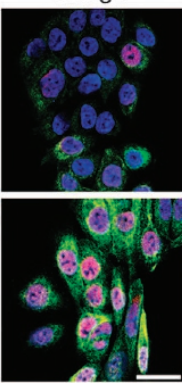

i
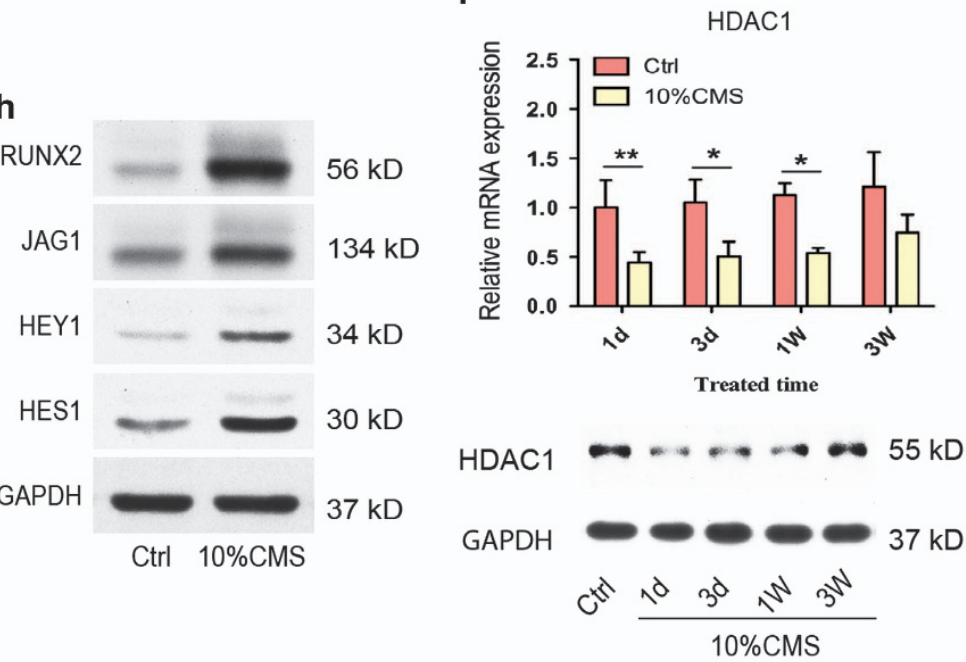

Figure 3 Cyclic mechanical loading could regulate osteogenic differentiation and NOTCH signaling in human BMSCs. (a) qRT-PCR analysis and (b) western blotting analysis of osteogenic differentiation markers ALP, COL1a1, and OCN in BMSCs after treatment with 10\% CMS for 3 weeks compared with static control cells. GAPDH was used as an internal control. (c) Representative images of ALP staining (including quantitative analysis) and (d) Alizarin red staining of BMSCs after treatment with $10 \%$ CMS for 3 weeks compared with static control cells. (e) Immunostaining of NICD (green) and RUNX2 (red) location in BMSCs after treatment with 10\% CMS for 3 weeks compared with static control cells. Scale bar, $50 \mu \mathrm{m}$. (f) qRT-PCR analysis of JAG1 expression in BMSCs after treatment with $10 \%$ CMS for 3 weeks compared with static control cells. (g) qRT-PCR analysis of NOTCH1 4, JAG1, JAG1, HES1, and HEY1 mRNA levels and (h) western blot analysis of BMSCs after treatment with $10 \%$ CMS for 3 weeks compared with static control cells. (i) qRT-PCR analysis and western blotting analysis of HDAC1 in BMSCs after treatment with $10 \% \mathrm{CMS}$ for 3 weeks compared with static control cells. All results are representative of at least three independent experiments. All the staining data were confirmed by three repeated tests. Data were mean $\pm S$.D., ${ }^{*} P<0.01,{ }^{* *} P<0.001$. All $P$-values are based on Student's t-test

(Figure 4j). Moreover, we then investigated whether the observed pro-osteogenic effects of HDAC1 inhibition were related to changes in histone acetylation in the promoter regions in human BMSCs. A ChIP analysis was performed using antibodies to pan-acetylated histone $\mathrm{H} 3$ and four designed primers for JAG1 promoters (Figure 5a). We identified a significant increase in the histone $\mathrm{H} 3$ acetylation level at the JAG1 promoter after CMS treatment for 3 weeks 
a

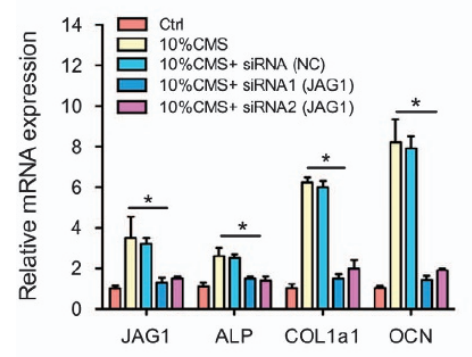

e

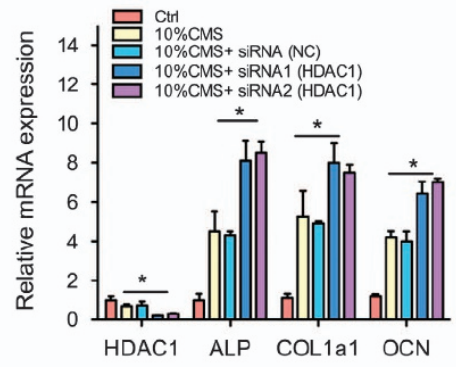

h

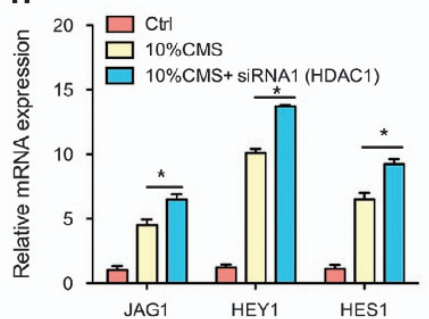

b

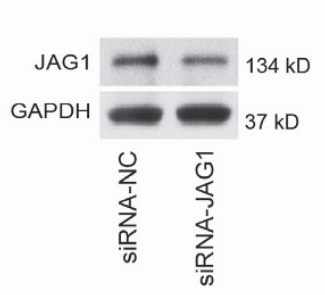

C

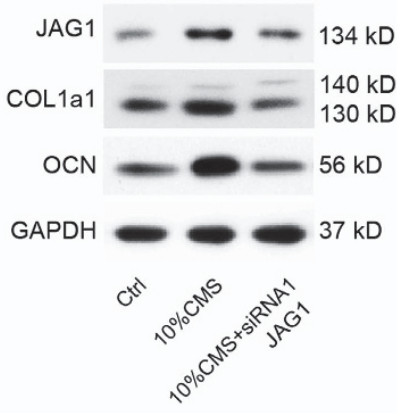

d

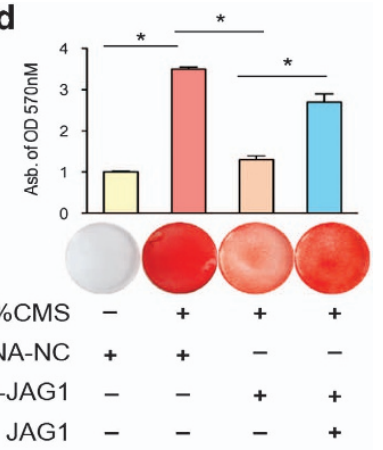

g
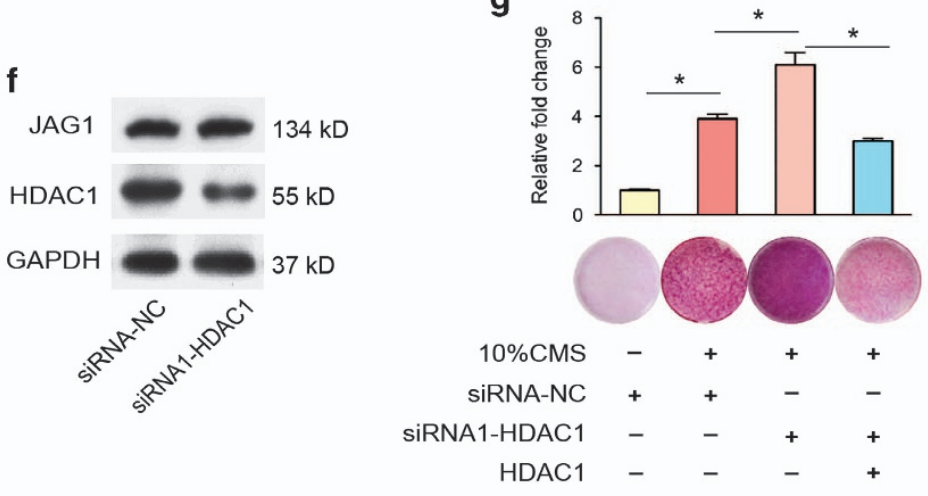

i

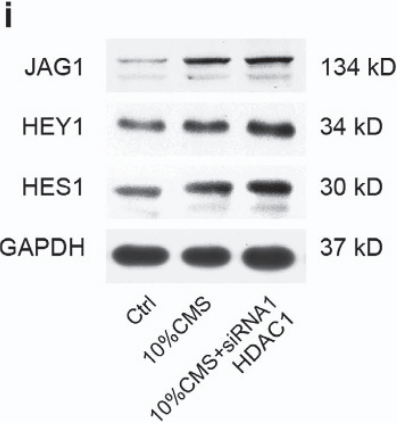

j

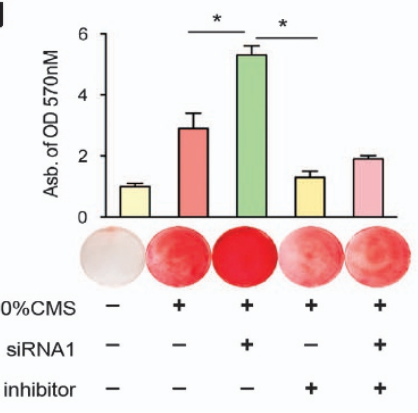

Figure 4 HDAC1 inhibition promoted CMS-induced osteogenic differentiation in human BMSCs. (a) qRT-PCR analysis and (b, c) western blotting analysis of JAG1, ALP, COL1a1, and OCN in BMSCs after transfected with specific siRNA-JAG1 or its corresponding scrambled control (siRNA-NC) under $10 \%$ CMS for 3 weeks. Two specific siRNAs for JAG1 were used, and then after the confirmation of knockdown efficiency, siRNA1 was used for the subsequent experiments. (d) Alizarin red staining of BMSCs after transfected with specific siRNA-JAG1 or its corresponding scrambled control or JAG1-overexpressing vector under 10\% CMS for 3 weeks. (e) qRT-PCR analysis of HDAC1, ALP, COL1a1, and OCN in BMSCs after transfected with specific siRNA-HDAC1 or its corresponding scrambled control (siRNA-NC) under 10\% CMS for 3 weeks. Two specific siRNAs for HDAC1 were used, and then after the confirmation of knockdown efficiency, siRNA1 was used for the subsequent experiments. (f) The knockdown efficiency of HDAC1specific siRNA (siRNA-HDAC1) was confirmed by comparison to a scrambled control siRNA (siRNA-NC). (g) Representative images of ALP staining (including quantitative analysis) of BMSCs after transfected with specific siRNA-HDAC1 or its corresponding scrambled control or HDAC1-overexpressing vector under $10 \%$ CMS for 3 weeks. (h) qRT-PCR analysis of JAG1, HES1, and HEY1 mRNA levels and (i) western blotting analysis of BMSCs after transfected with specific siRNA1-HDAC1 under 10\% CMS for 3 weeks. (j) Alizarin red staining of BMSCs after co-transfected with specific siRNA-HDAC1 with inhibitor of Notch signaling transduction (10 nM RO4929097) under 10\% CMS for 3 weeks. GAPDH was used as an internal control. All results are representative of at least three independent experiments. All the staining data were confirmed by three repeated tests. Data were mean \pm S.D., ${ }^{*} P<0.01$. All $P$-values are based on Student's $t$-test

(Figures $5 \mathrm{~b}$ and $\mathrm{c}$ ). HDAC1 inhibition promoted the elevated $\mathrm{H} 3$ acetylation level at the JAG1 promoter (Figure $5 \mathrm{~d}$ ).

Second, we overexpressed HDAC1 in human BMSCs (Figure 5e) and found that this significantly reduced ALP and COL1a1 transcription and the level of the late osteoblast marker OCN under mechanical stimulation conditions (Figure 5f). ALP assays and Alizarin red staining for mineralized deposits showed that HDAC1 overexpression significantly abrogated the CMS-induced increase in ALP activity and mineralized deposits (Figures $5 \mathrm{~g}$ and $\mathrm{h}$ ). Furthermore, the CMS-induced expression of JAG1, HES1, and HEY1 were also blocked by HDAC1 overexpression (Figures $5 \mathrm{i}$ and $\mathrm{j}$ ). Consistent with the above results, the CMS-enhanced histone $\mathrm{H} 3$ acetylation level at the JAG1 promoter was apparently decreased by the induction of HDAC1 overexpression (Figure 5k). Overall, these results 
a

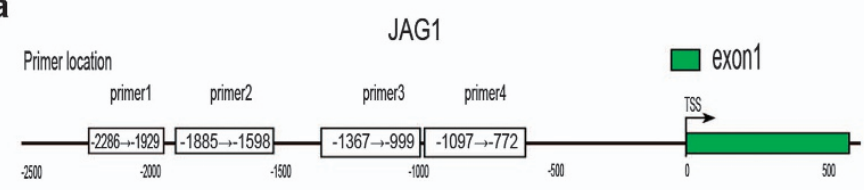

C

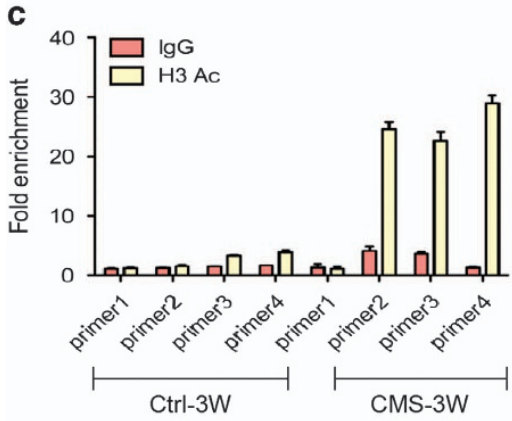

d

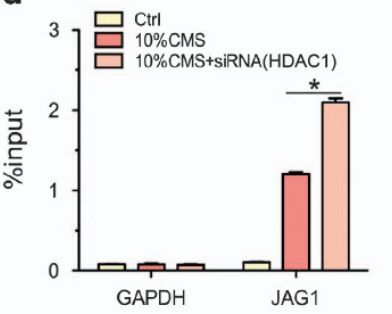

e

HDAC1 $=55 \mathrm{kD}$

GAPDH

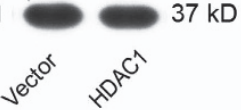

f

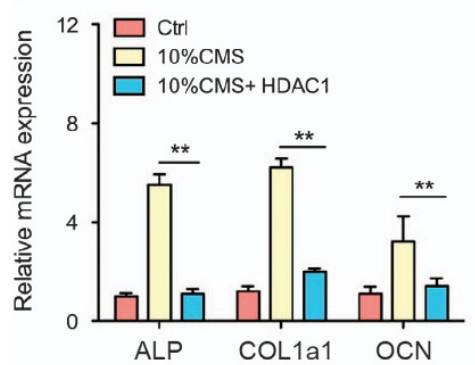

g

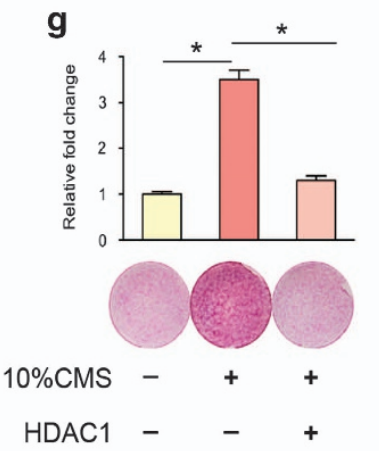

b

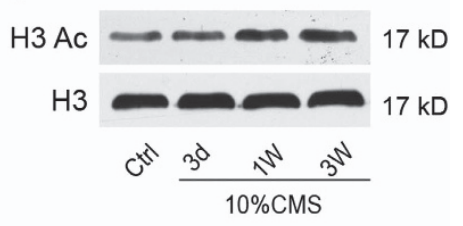

i

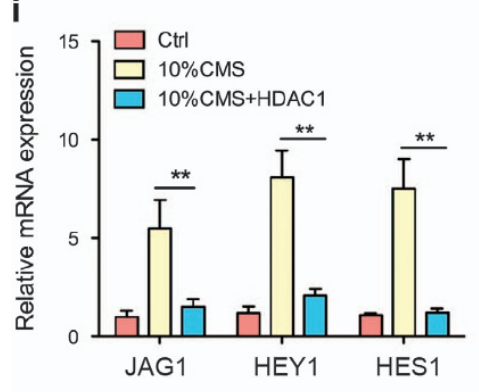

j

h

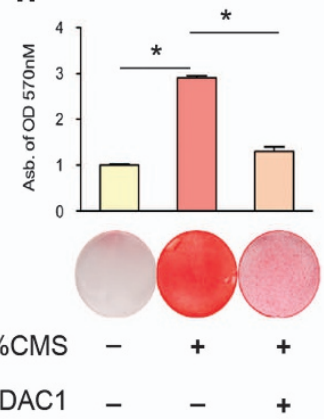

k

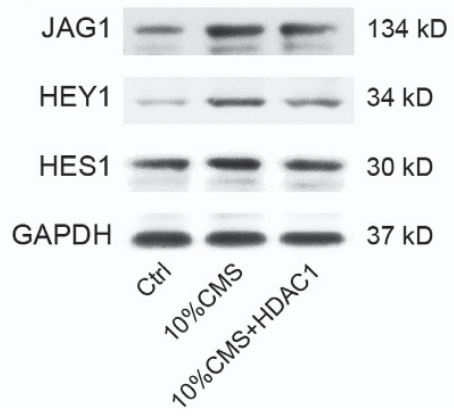

Figure 5 HDAC1 overexpression blocked CMS-induced osteogenic differentiation in human BMSCs. (a) Scheme of primers' location in the 5'-flank promoter region of JAG1 gene. The transcriptional start site (TSS) is indicated as +1 . (b) Western blotting analysis revealed a increase in acetylation of histone $\mathrm{H} 3(\mathrm{H} 3$ ) in $\mathrm{BMSCs}$ after treatment with $10 \%$ CMS for 3 weeks compared with static control cells. (c) ChIP-qPCR analysis of H3 acetylation modification in different JAG1 promoter regions in BMSCs after treatment with $10 \%$ CMS for 3 weeks compared with static control cells. (d) ChIP-qPCR assay on GAPDH and JAG1 promoters. ChIP analysis revealed that there was a significant increase in histone H3 acetylation at JAG1 promoters after siRNA-HDAC1 treatment in BMSCs. (e) The efficiency of HDAC1 overexpression was confirmed by comparison to a empty vector. (f) qRTPCR analysis of osteogenic differentiation markers ALP, COL1a1, and OCN. (g) ALP staining (including quantitative analysis) and (h) Alizarin red staining (including quantitative analysis) in BMSCs after transfected with HDAC1 overexpression or its corresponding negative control under $10 \% \mathrm{CMS}$ for 3 weeks. (i) qRT-PCR analysis of JAG1, HES1, and HEY1 mRNA levels and (j) western blotting analysis of BMSCs after transfected with HDAC1 overexpression under $10 \% \mathrm{CMS}$ for 3 weeks. (k) ChIP-qPCR analysis of H3 acetylation modification in different JAG1 promoter regions in BMSCs after transfected with HDAC1 overexpression under $10 \% \mathrm{CMS}$ for 3 weeks. GAPDH was used as an internal control. All results are representative of at least three independent experiments. All the staining data were confirmed by three repeated tests. Data were mean $\pm S . D .{ }^{*} P<0.01$. All $P$-values are based on Student's t-test

confirm that CMS can induce osteogenic differentiation of BMSCs by activating pro-osteogenic JAG1-Notch signaling, which is facilitated by increased histone $\mathrm{H} 3$ acetylation levels.
Inhibition of HDAC1 rescued the decrease of bone formation in the $\mathrm{HU}$ mouse model. To investigate the function of HDAC1 in vivo, we used a $\mathrm{HU}$ mouse model. MicroCT showed that mechanical unloading-induced bone 
a

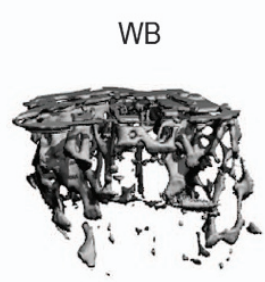

HU

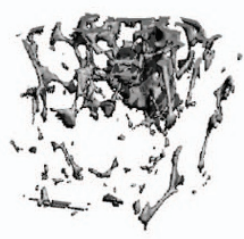

HU+inhibitor(HDAC1)

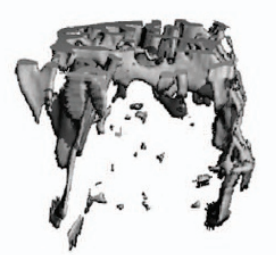

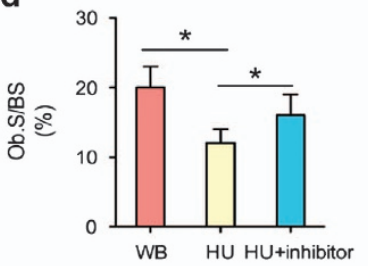
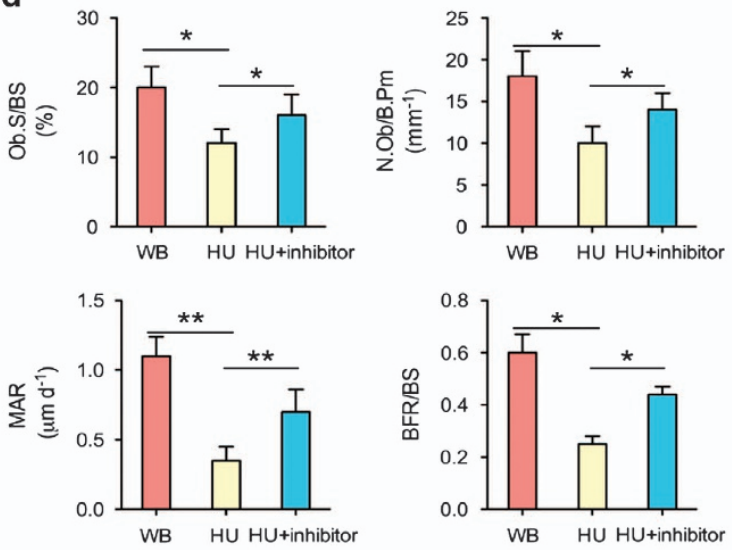

e WB HU

HU

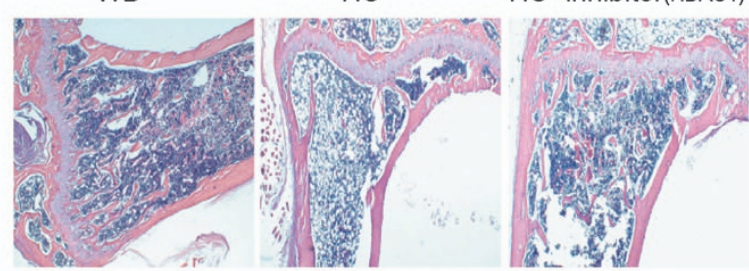

f

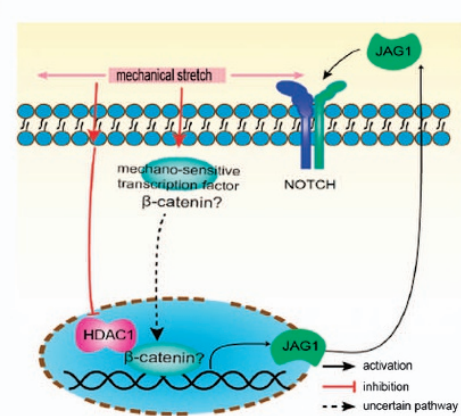

Figure 6 Inhibition of HDAC1 rescued the decrease of bone formation in vivo. (a) Representative microCT reconstructive images of tibial plateau in WB, HU, and HU+HDAC1 inhibitor mice. $n=6$. (b) Three-dimensional microstructural parameters of tibial plateau in WB, HU, and HU+HDAC1 inhibitor mice. (c) Representative images showing new bone formation assessed by double calcein labeling in each group. $n=4$. Scale bars, $50 \mu \mathrm{m}$. (d) Histomorphometric analysis of bone formation-related parameters (Ob.S/BS, MAR, $\mathrm{BFR}$, and N.Ob/B.Pm) in WB, HU, and HU+HDAC1 inhibitor mice. (e) Representative hematoxylin-eosin staining images of tibial plateau showing bone volume in each group. $n=6$. (f) Schematic diagram of the role of HDAC1 in regulating MSC differentiation and bone formation under mechanical stimulation. Data were mean \pm S.D., ${ }^{\star} P<0.01$. All $P$-values are based on Student's $t$-test

loss was partly rescued by HDAC1 inhibition (valproic acid) (Figure 6a), and bone volume analysis revealed that the bone volume-related parameters were partly increased by HDAC1 inhibition (Figure 6b). Similarly, the assessment of bone formation indicated by green fluorescent calcein showed that the mechanical unloading-induced decrease in bone formation was rescued by HDAC1 inhibition (Figures $6 c$ and d). Furthermore, a histological assessment after 28 days of $\mathrm{HU}$ demonstrated that, compared with staining in the WB group, the bone staining of the HU group was significantly decreased, with intensified adipose tissue staining. The overall area of stained bone was increased in the HU group treated with an HDAC1 inhibitor, which indicates that the impaired development of new bone was rescued by HDAC1 inhibition (Figure 6e). Taken together, these results indicate that osteogenic differentiation and Notch signaling are impaired in the BMSCs of patients with osteoporosis and in $\mathrm{HU}$ mice. In addition, mechanical stimulation promotes the osteogenic differentiation of BMSCs by, at least in part, activating JAG1-mediated pro-osteogenic Notch signaling and reducing the expression of HDAC1. Finally, the therapeutic inhibition of HDAC1 was able to partly counteract the bone loss observed in HU mice (Figure 6f).

\section{Discussion}

Understanding how MSCs sense and respond to applied forces is an area of intense research. Numerous cell membrane proteins have been identified in mechano-sensing mechanisms. ${ }^{25-27}$ Cadherins, which bind cells to adjacent cells, have also been found to anchor themselves by forming complexes with catenins, which bind directly to the 
cytoskeleton. Mechanical loading inhibits the binding of $\beta$-catenin to $E$-cadherin and increases the cytoplasmic pool of $\beta$-catenin. ${ }^{28}$ The decrease of $\beta$-catenin binding is coupled with Akt and GSK3 $\beta$ activation. Therefore, cadherins may serve as mechano-sensors and may be a promising target for future mechanical loading studies. The expression of Notch, a cell-surface receptor that transduces short-range signals by interacting with transmembrane ligands such as Delta (termed Delta-like in humans) and Serrate (termed Jagged in humans) on neighboring cells, had not yet been studied under mechanical stimulation conditions. Notch signaling is a key mechanism in the control of stem cell differentiation and embryogenesis. ${ }^{29}$ Notch signaling components, especially JAG1 and NOTCH-2, are upregulated during both endochondral and intramembranous bone regeneration. ${ }^{30}$ JAG1 is variably mutated in Alagille syndrome patients with skeletal defects and poor bone healing. ${ }^{31}$ In addition, JAG1 has been identified as a gene associated with osteoporosis in a GWAS analysis, and a recently identified Jag1-null mutation is responsible for the development of osteogenesis imperfecta. ${ }^{23}$ Studies have also found that JAG1-activated Notch signaling is sufficient to induce human MSC osteogenesis, increasing osteogenic marker genes, such as ALP and BSP, and enhancing ALP activity and tissue mineralization. ${ }^{21}$

Despite the clinical and genetic evidence suggesting that JAG1 may positively regulate bone mass in humans, there is a paucity of data from studies exploring the role of JAG1 in human osteogenesis under mechanical loading. For the first time, our results show that Jag1 and Notch signaling were decreased in the BMSCs of hindlimb-unloaded mice and that JAG1-activated NOTCH signaling in human BMSCs was upregulated by CMS in vitro. Furthermore, JAG1 and Notch signaling had an important role in mediating the mechanical-triggered osteogenic differentiation of BMSCs, which was blocked by inhibitors of the $\mathrm{NOTCH}$ signaling pathways. However, we also observed that CMS modulated JAG1 expression by modifying the histone acetylation status of JAG1, as the inhibition of HDAC1 only partially rescued the mechanical unloading-induced osteoporosis in mice. This suggests that CMS may also directly induce JAG1 expression by activating mechano-sensitive transcription factors, such as $\beta$-catenin, and change the epigenetic modifications of the JAG1 promoter region to promote its transcription. NOTCH signaling and JAG1 expression were both impaired because of the loss of mechanical loading, but they may also be affected by the loss of miscellaneous biochemical or spatial structural signals that occurs in osteoporosis in vivo. This topic deserves in-depth studies to determine how BMSCs orchestrate the biochemical and mechanical signals involved in this process and which signal is the main factor in controlling osteogenic differentiation in vivo.

Stem cell differentiation is extremely sensitive to epigenetic changes. The application of epigenetic regulators, such as inhibitors of histone-modification enzymes, may be valuable for stem cell-based interventions. ${ }^{32}$ HDAC inhibitors have been demonstrated to enhance osteogenic differentiation in vitro and new bone formation in vivo. ${ }^{19}$ Previous studies have shown that the suppression of HDAC activity with HDAC inhibitors accelerates osteogenesis by inducing osteoblast marker genes, including osteopontin and ALP. Moreover, the osteogenesis-promoting effects of VPA on the expression of bone matrix markers are related to changes in histone acetylation in the promoter regions. ${ }^{33}$ It has also been shown that mechanical cues could directly induce changes in epigenetic modifications. ${ }^{34}$ In vascular endothelial cells (ECs), hemodynamic force-induced histone modifications have been extensively studied in recent years. Shear stress can modulate chromatin remodeling on histone $\mathrm{H} 3$ and $\mathrm{H} 4$, resulting in eNOS being regulated by chromatin-based epigenetic mechanisms at the transcriptional level. ${ }^{35}$ Zeng et al. ${ }^{36}$ demonstrated that laminar flow increased the activity of HDACs and the association of p53 with HDAC1, leading to the deacetylation of p53 in ECs. Lee et al. ${ }^{37}$ utilized HDACspecific siRNAs and found that class I HDAC1/2/3, but not class II HDAC4/7, modulated oscillatory flow-induced cell proliferation. However, the potential of the mechanical environment to regulate DNA methylation or histone modifications has seldom been examined in BMSCs, which is fundamental to understanding stem cell mechanobiology. Arnsdorf et al. ${ }^{38}$ demonstrated that mechanical stimulation altered the epigenetic state of osteogenic genes (Ocn, Opn, and Col1) by reducing DNA methylation and showed that this was associated with an increase in expression. Zuo et al. ${ }^{13}$ found that miR-103a is a mechano-sensitive miRNA that regulates osteoblast differentiation via directly targeting Runx2. For the first time, we found that mechanical loading downregulated HDAC1 and, therefore, facilitated JAG1 expression and the osteogenesis of BMSCs. Furthermore, we also found increased Hdac1 expression and downregulated Jag1-Notch signaling in a mechanical unloading-induced osteoporotic mouse model. The inhibition of Hdac1 by a specific siRNA or small-molecule inhibitor promoted osteogenesis and rescued bone loss in mechanically unloaded mice. Consistent with the above-mentioned reported results, our results confirmed that mechanical cues can directly modulate the epigenetic modification status of osteogenic genes and, therefore, promote the osteogenic differentiation of BMSCs.

Several studies have indicated that mechanical force has an important role in regulating cell growth and proliferation, and an appropriate mechanical stretch treatment could promote the proliferative capacity of BMSCs. Song et al. ${ }^{39}$ found that the proliferation of rat bone marrow mesenchymal stem cells was significantly elevated after exposure to a $1-\mathrm{Hz}$ stretch stimulation within 15-60 min at an 8\% strain. However, Zuo et al. ${ }^{13}$ found that $8 \% \mathrm{CMS}$ had no significant influence on cell viability during $24-72 \mathrm{~h}$. The effect of long-term ( $>3$ weeks) continuous mechanical stretching on the proliferation of MSCs has not been previously studied. Luu et al. ${ }^{40}$ found that 6 weeks of low-magnitude mechanical signals $(0.2 \mathrm{~g}, 90-\mathrm{Hz}$ signal applied for $15 \mathrm{~min} /$ day, 5 day/week) increased the overall marrow-based stem cell population by $37 \%$ and the number of MSCs by $46 \%$. Concomitant with the increase in stem cell number, the differentiation potential of MSCs in the bone marrow was biased toward osteogenic and against adipogenic differentiation. In our study, we found that without mechanical loading, the proliferation of BMSCs was unchanged after 3 weeks. There are many studies that have shown mechanical stimulation to have no effect on cell proliferation $^{8,41}$ or to reduce MSC proliferation. , $^{2,42,43}$ These 
mixed findings can most likely be explained by the diversity of conditions in the experiments, including the specific mechanical stimulation, MSC species, and culture media used, as well as the wide range of loading parameters used.

\section{Conclusion}

In summary, our study provides new findings that mechanical stimulation orchestrates gene expression for the osteogenic differentiation of BMSCs via directly regulating HDAC1. HDAC1 functions through inhibiting its direct target, JAG1, which is the master regulator of osteogenesis, at the transcription level. Understanding the molecular mechanisms of epigenetic modifiers such as HDACs in regulating MSC lineage determination under mechanical stimulation is pivotal for understanding bone cell differentiation and diseases. These findings not only provide new insights into mechanoresponse signaling pathways but also raise intriguing possibilities for the use of HDAC modulators to regulate bone formation in regenerative medicine. We anticipate that our study will provide a foundation for future investigations on the development of gene therapies for treating human bone remodeling disorders related to mechanical loading, such as osteoporosis.

\section{Materials and Methods \\ Cell culture. Bone marrow cells from the tibias and femurs of mice and the posterior iliac crests of healthy adult human donors (17-35 years of age), collected with informed consent, were flushed out with $\alpha$-Minimum Essential Medium $(\alpha$-MEM, Hyclone, Logan, UT, USA) and cultured in growth medium ( $\alpha$-MEM with $10 \%$ fetal bovine serum (Gibco by Invitrogen, Carlsbad, CA, USA) with $1 \%$ penicillin and streptomycin (Hyclone)) at $37^{\circ}$ in the presence of $5 \% \mathrm{CO}_{2}$ following the lysis of red blood cells. Non-adherent cells were removed by replacing the medium after 3 days. The attached BMSCs were used for experiments at passages 3-5. \\ For the transfection of siRNA oligos, the cells in the culture medium were transfected using Lipofectamine 2000 transfection reagent (Invitrogen, Carlsbad, CA, USA), which was used according to the manufacturer's instructions. The siRNA was transfected at a concentration of $50 \mathrm{nM}$. The siRNA sequences used in this study were as follows: for JAG1, siRNA1: 5'-AGGCTGCGCATAATCATAATA-3', siRNA2: 5'-GGCTGCGCATAATCATAATAA-3'; and for HDAC1, siRNA1: 5'-GGAGGAAAGTCT GTTACTACT-3', siRNA2: 5'-GAGGAAAGTCTGTTACTACTA-3'.}

Hindlimb unloading (HU) in mice. Six-month-old male C57BL/6J mice were purchased from Shanghai SLAC Laboratory Animal Co. Ltd, Shanghai, China. The animals were suspended from the hindlimbs for a period of 28 days, as previously described. ${ }^{13}$ All of the experimental procedures were approved by the Committees of Animal Ethics and Experimental Safety of Shanghai Ninth People's Hospital.

Retroviral transduction overexpression studies. Human JAG1 and HDAC1 genes were ligated into pRUF-IRES-GFP using PCR primers to amplify the coding region. The pRUF- IRES-GFP and pRUF-IRES-GFP-JAG1 and HDAC1 constructs were transfected into the HEK 293T viral packaging cell line together with the Pol and GAG protein (PGP) and vesicular stomatitis virus G-protein (VSVG) (viral envelope proteins, SBI System Biosciences, Mountain View, CA, USA). The viral supernatant was used for the infection of MSCs as previously described. ${ }^{44}$ Stable lines were generated by sorting for GFP-positive cells using fluorescenceactivated cell sorting.

Microarray analysis and Gene Set Enrichment Analysis (GSEA). The microarrays for human BMSCs of elderly individuals or patients with osteoporosis (GSE35959) were obtained from the GEO database. To identify genes that are differentially expressed between normal and osteoporosis donors, the spot intensity data of all relevant samples were analyzed using GeneSight-Lite 4.1.6 (BioDiscovery, El Segundo, CA, USA). Normalization of the expression profiles was performed by dividing values by the mean signal of each array representing a single sample. The resulting data were visualized using the Multiple Experiment Viewer application (Boston, MA, USA). Gene sets from the complete C2 curated gene sets and C5 GO gene sets, downloaded from Molecular Signatures Database (MSigDB), were tested for enrichment against the human osteoporosis phenotype based on the GSEA method. ${ }^{45}$ The complete series of human BMSCs in samples from four elderly donors (GSM878100, GSM878101, GSM878102, and GSM878103) and four osteoporosis donors (GSM878104, GSM878105, GSM878106, and GSM878107) were used for this analysis. Genes were sorted according to the value of the $t$-statistic computed against the human BMSC 'Normal versus Osteoporosis' phenotype, with genes upregulated in the 'Normal' class at the leftend of the list and genes upregulated in the 'Osteoporosis' class at the right-end of the list; the cell cycle checkpoint, Notch pathway and skeletal development genes were located within the sorted list, and their position was determined to be significantly skewed toward the 'Normal' end of the sorted list based on a weighted Kolmogorov-Smirnoff test. The resulting heat map and the intensity data were partly inspected for genes differentially expressed between the BMSCs of normal donors and those with osteoporosis.

Cyclic mechanical stretch application. BMSCs were plated at a density of $5 \times 10^{5} \mathrm{cell} / \mathrm{s} / \mathrm{cm}^{2}$ (if not mentioned) in $1 \mathrm{ml}$ of medium on six-well flexible silicone rubber BioFlex plates coated with collagen type I (Flexcell International Corporation, Hillsborough, NC, USA). Cells were cultured for $24 \mathrm{~h}$ to reach $50-60 \%$ confluency before mechanical tension was applied, which guaranteed sufficient space for cell proliferation and an adequate number of cells for the following experiments. Cyclic mechanical stretch (CMS) with a $0.5-\mathrm{Hz}$ sinusoidal curve at $10 \%$ elongation was applied using an FX-5000 T Flexercell Tension Plus unit (Flexcell International Corporation). The cultures were incubated in a humidified atmosphere at $37^{\circ} \mathrm{C}$ and $5 \% \mathrm{CO}_{2}$ during the stretching. Cells were harvested immediately after the application of CMS stimulation was completed. Control cells were cultured on the same plates in the same incubator but were not subjected to stretching.

Proliferation assays. For the proliferation assays, BMSCs were seeded at a density of 4000 cells/well in 96 -well plates, and cell proliferation was monitored after the indicated time points using an MTT (3-(4,5-dimethylthiazol-2-yl)-2,5-diphenyltetrazolium bromide) assay.

ALP staining. ALP staining was performed on cultured cells. The cell layer was rinsed with phosphate-buffered saline (PBS) three times, followed by fixation in $4 \%$ paraformaldehyde for $10 \mathrm{~min}$ at room temperature. The cells were then incubated with buffer containing $0.1 \%$ naphthol AS-TR phosphate and $2 \%$ fast violet $\mathrm{B}$ (Sigma-Aldrich, St Louis, MO, USA). After incubation for $1 \mathrm{~h}$ at $37^{\circ} \mathrm{C}$, the cell layer was washed with deionized water.

Alizarin red staining. Cells were fixed in $70 \%$ ice-cold ethanol for $1 \mathrm{~h}$ and rinsed with double-distilled $\mathrm{H}_{2} \mathrm{O}\left(\mathrm{ddH}_{2} \mathrm{O}\right)$. Cells were then stained with $40 \mathrm{mM}$ Alizarin red S (Sigma, St. Louis, MO, USA), pH 4.0, for 15 min with gentle agitation. After staining, cells were rinsed five times with $\mathrm{ddH}_{2} \mathrm{O}$. For the quantitative assessment of the degree of mineralization, the red stain was eluted by $10 \%(\mathrm{w} / \mathrm{v})$ cetylpyridinium chloride (Sigma-Aldrich) for $1 \mathrm{~h}$ and quantified via spectrophotometric absorbance measurements of optical density at $570 \mathrm{~nm}$.

RNA purification and quantitative real-time PCR (qRT-PCR). The total RNA of cells was isolated using TRIzol reagent (Invitrogen) according to the manufacturer's instructions. After the reverse transcription reaction, RT-PCR was performed with an ABI 7900HT system using SYBR Premix (Takara, Dalian, China) according to the manufacturer's instructions. The conditions of the RT-PCR were as follows: denaturation at $95^{\circ} \mathrm{C}$ for $10 \mathrm{~s}, 40$ cycles at $95^{\circ} \mathrm{C}$ for $10 \mathrm{~s}$, and $60^{\circ} \mathrm{C}$ for $30 \mathrm{~s}$. A dissociation stage was added at the end of the amplification procedure. No nonspecific amplification was observed, as determined using the dissociation curve. Glyceraldehyde 3-phosphate dehydrogenase (GAPDH) was used as an internal control. The data were analyzed using the comparison $\mathrm{Ct}\left(2^{-\Delta \Delta \mathrm{Ct}}\right)$ method and expressed as the fold change relative to the respective control. Each sample was analyzed in triplicate. The primer sequences used in this study were as follows: GAPDH: forward, 5'-CCTCTGACTTCAACAGCGAC-3'; reverse, 5'-TCCTCTTGTG CTCTTGCTGG-3'; ALP: forward, 5'-GAGTCGGACGTGTACCGGA-3'; reverse, $5^{\prime}$-TGCCACTCCCACATTTGTCAC-3'; runt-related transcription factor 2 (RUNX2): forward, 5'-GCCTTCAAGGTGGTAGCCC-3'; reverse, 5'-CGTTACCCGCCATGACA GTA-3'; COL1a1: forward, 5'-CAGCCGCTTCACCTACAGC-3'; reverse, 5'-TTTTGT 
ATTCAATCACTGTCTTGCC-3'; OCN: forward, 5'-GAAGCCCAGCGGTGCA-3'; reverse, 5'-CACTACCTCGCTGCCCTCC-3'; OSX: forward, 5'-CCCTTCTCAAGCA CCAATGG-3'; reverse, 5'-AAGGGTGGGTAGTCATTTGCATA-3'; fatty acid-binding protein-4 (aP2): forward, 5'-AAATCACCGCAGACGACA-3'; reverse, $5^{\prime}$-CACATTC CACCACCAGCT-3'; glucose transporter type 4 (Glut4): forward, $5^{\prime}$-CTTGGCTCCC TTCAGTTTG-3'; reverse, $5^{\prime}$-TGCCTTGTGGGATGGAAT-3'; hes family bHLH transcription factor 1 (HES1): forward, 5'-TCAACACGACACCGGATAAAC-3'; r1verse, 5'-GCCGCGAGCTATCTTTCTTCA-3'; hes-related family bHLH transcription factor with YRPW motif 1 (HEY1): forward, 5'-GTTCGGCTCTAGGTTCCATGT-3'; reverse, 5'-CGTCGGCGCTTCTCAATTATTC-3'; for human: JAG1: forward, 5'-GTCCATGCA GAACGTGAACG-3'; reverse, 5'-GCGGGACTGATACTCCTTGA-3'; JAG2: forward 5'-TGGGCGGCAACTCCTTCTA-3'; reverse, 5'-GCCTCCACGATGAGGGTAAA-3'; NOTCH1: forward, 5'-GAGGCGTGGCAGACTATGC-3'; reverse, 5'-CTTGTACTCC GTCAGCGTGA-3'; NOTCH2: forward, 5'-CCTTCCACTGTGAGTGTCTGA-3'; reverse, 5'-AGGTAGCATCATTCTGGCAGG-3'; NOTCH3: forward, 5'-CGTGGCTT CTTTCTACTGTGC-3'; reverse, 5'-CGTTCACCGGATTTGTGTCAC-3'; NOTCH4: forward, 5'-TGTGAACGTGATGTCAACGAG-3'; reverse, 5'-ACAGTCTGGGCCTAT GAAACC-3'; HDAC1: forward, 5'-CTACTACGACGGGGATGTTGG-3'; reverse, 5'-GAGTCATGCGGATTCGGTGAG-3'; for mice: Notch1: forward, 5'-GATGGCCTC AATGGGTACAAG-3'; reverse, 5'-TCGTTGTTGTTGATGTCACAGT-3'; Notch2: forward, 5'-GAGAAAAACCGCTGTCAGAATGG-3'; reverse, 5' -GGTGGAGTATTGG CAGTCCTC-3'; Notch3: forward, 5'-AGTGCCGATCTGGTACAACTT-3'; reverse, 5'-CACTACGGGGTTCTCACACA-3'; Notch4: forward, 5'-CCCCGGAGCATTCTT CTGC-3'; reverse, 5'-AGTCCAGCCCTCATCACACA-3'; Jag1: forward, 5'-ATGCAG AACGTGAATGGAGAG-3'; reverse, 5'-GCGGGACTGATACTCCTTGAG-3'; Jag2: forward, 5'-TTCTGTGACGAGTGTGTCCC-3'; reverse, 5'-GCGCAGAGGTATTGGT CAGG-3'; Hdac1: forward, 5'-TGAAGCCTCACCGAATCCG-3'; reverse, 5'-GGGCG AATAGAACGCAGGA-3'; Hdac2: forward, 5'-GGAGGAGGCTACACAATCCG-3'; reverse, 5'-TCTGGAGTGTTCTGGTTTGTCA-3'; Hdac3: forward, 5'-GCCAAGAC CGTGGCGTATT-3'; reverse, 5'-GTCCAGCTCCATAGTGGAAGT-3'; and Hdac5: forward, 5'-AGCACCGAGGTAAAGCTGAG-3'; reverse, 5'-GAACTCTGGTCCAAAG AAGCG-3'

Western blotting analysis. For the western blotting analysis, cells were lysed on ice for $30 \mathrm{~min}$ in a lysis buffer containing $50 \mathrm{mM}$ Tris- $\mathrm{HCl}(\mathrm{pH} 7.4)$, $150 \mathrm{mM} \mathrm{NaCl}, 1 \%$ Nonidet P-40, and $0.1 \%$ SDS supplemented with protease inhibitors $(10 \mathrm{mg} / \mathrm{ml}$ leupeptin, $10 \mathrm{mg} / \mathrm{ml}$ pepstatin $\mathrm{A}$, and $10 \mathrm{mg} / \mathrm{ml}$ aprotinin). Protein fractions were collected by centrifugation at $15000 \mathrm{~g}$ at $4{ }^{\circ} \mathrm{C}$ for $10 \mathrm{~min}$ and then subjected to $10 \%$ SDS-PAGE and transferred to polyvinylidene difluoride membranes. The membranes were blocked with 5\% BSA and incubated with specific antibodies overnight at $4{ }^{\circ} \mathrm{C}$. A horseradish peroxidase-labeled secondary antibody was added and visualized using an enhanced chemiluminescence detection system (Millipore, Billerica, MA, USA) as recommended by the manufacturer. We used the following primary antibodies to determine the concentrations of proteins in the lysates: anti-human RUNX2 rabbit mAb, anti-JAG1, HEY1 mAb (1:1000, Abcam, Cambridge, UK), anti-NOTCH1, HES1 mAb (1:1000, Cell Signaling Technology, Inc., Danvers, MA, USA), GAPDH rabbit mAb (1:1000, Cell Signaling Technology, Inc.), anti-HDAC1, HDAC2, HDAC3, HDAC5 mAb (1 : 1000, Abcam), anti-COL1a1, OCN (1:500, Abcam), and anti-AcH3 antibody (1:1000, Millipore, Bedford, MA, USA)).

Immunofluorescence. BMSCs cultured in six-well plates were fixed with $4 \%$ PFA in PBS for 20 min at room temperature. After washing in PBS, samples were permeabilized with $0.5 \%$ Triton X-100 for $5 \mathrm{~min}$ and blocked with $5 \%$ BSA for $60 \mathrm{~min}$. An incubation with primary anti-NICD and anti-RUNX2 antibodies (Abcam) was performed overnight at $4{ }^{\circ} \mathrm{C}$. The primary antibodies were detected using FITC or PE-conjugated anti-mouse IgG secondary antibodies. After the final wash, the nuclei were counterstained by adding a $2-\mathrm{mg} / \mathrm{ml}$ solution of $4^{\prime}, 6$-diamidino-2phenylindole (Sigma-Aldrich) in PBS for $10 \mathrm{~min}$ before imaging. Cells were visualized using a confocal microscope (Leica, Solms, Germany).

Bone histomorphometric analyses. We measured the structure of the tibial plateau with a SCANCO Medical $\mu \mathrm{CT} 40$ scanner. The images were analyzed using the SCANCO evaluation software to perform segmentation, conduct a threedimensional morphometric analysis, and determine the density and distance parameters (SCANCO Medical AG, Zurich, Switzerland). The three-dimensional structural parameters analyzed included the following: TV (total tissue volume, containing both trabecular and cortical bone), BV/TV (trabecular bone volume per tissue volume), Tb.Th (trabecular thickness), Tb.Sp (trabecular separation), and SMI (structure model index). For the assessment of new bone formation, we injected green fluorescent calcein (Sigma; $5 \mathrm{mg} / \mathrm{kg}$ body weight) into the mice on days 7 and 2 before killing. Bone histomorphometric analyses for OB number per bone surface (Ob.S/BS), OB number per bone perimeter (N.Ob/B.Pm), bone formation rate/bone surface (BFR/BS), and mineral apposition rate (MAR) were performed using the professional image analysis software (Image J; NIH, Bethesda, MD, USA) under fluorescence microscopy (Leica, Q500MC). The bone histomorphometric parameters were calculated and expressed according to the standardized nomenclature for bone histomorphometry.

Chromatin immunoprecipitation (ChIP) assay. ChIP assays were performed using an EZ ChIP Chromatin Immunoprecipitation Kit (Millipore, Upstate, NY, USA) according to the manufacturer's instructions. Briefly, cells cultured under the previously indicated conditions were fixed in $1 \%$ formaldehyde/PBS for $10 \mathrm{~min}$ at room temperature. After two washes with PBS, cells were resuspended in $0.5 \mathrm{ml}$ of lysis buffer containing a protease inhibitor cocktail before sonication. DNA fragments from the soluble chromatin preparations were $400-800$ bp in length. Immunoprecipitation was carried out overnight with purified anti-AcH3 antibody (Millipore, Bedford, MA, USA) or normal mouse lgG as a negative control. Protein $A / G$ agarose was used to pulldown the antigen-antibody compounds and then washed four times with washing buffers. The DNA-protein crosslinks were reversed with $5 \mathrm{M}$ $\mathrm{NaCl}$ at $65^{\circ} \mathrm{C}$ for $6 \mathrm{~h}$, and DNA from each sample was purified. PCR was performed using $2 \mu \mathrm{l}$ DNA samples with the following primers: JAG1 primer 1: forward, 5'-TTCTAGGTGAAGCCAGGTGGAG-3'; reverse, 5'-AATACAAAAATTAGC TGGGCGTG-3'; primer 2: forward, 5'-AATCTCTTGACCTCGTGATCCACC-3'; reverse, 5'-AGCGACAACCTGGGTGTTTCAAT-3'; primer 3: forward, 5'-GAATGAT GAGATTTGGCACTGAA-3'; reverse, 5'-CTGGTCATAATCAAGGTCGAAGA-3'; and primer 4: forward, 5'-TATAAAGGTCCCCTCAAATGCAAC-3'; reverse, 5'-AGATGCT GGTGGGCTTGGAC-3'.

Therapeutic inhibition of HDAC1 in HU mice. Six-month-old C57BL/6J mice received tail-vein injections of valproic acid ( $\mathrm{HU}$ +inhibitor group, valproic acid was purchased from Selleckchem (Shanghai, China), $50 \mathrm{mg} / \mathrm{kg}$ body weight, $0.2 \mathrm{ml}$ per injection) twice a week for 2 weeks or no treatment (HU group). The mice were subjected to $\mathrm{HU}$ via tail suspension for 27 days and were then killed. Tissues were harvested, and measurements of bone formation in the tissues were performed.

Statistical analysis. The data are presented as the mean \pm S.D. ( $n$ is the number of tissue preparations, cells, or experimental replicates). For comparing groups of data, a two-tailed Student's $t$-test was used. A value of $P<0.05$ was considered to be statistically significant.

\section{Conflict of Interest}

The authors declare no conflict of interest.

Acknowledgements. This work was supported by grants from National Key Project of Scientific and Technical Supporting Programs funded by Ministry of Science \& Technology of China (No: 2012BAl11B03), National Key Specialty of Clinical Plastic Surgery Project, and National Natural Science Foundation of China (No. 81190133, 81572123).

\section{Author contributions}

JW, CDW, and QFL: conception and design; JW, CDW, YFZ, SZS, NZ, and WXT: experiments and/or data analysis; QFL: clinical consultancy; WXT: intellectual input and supervision; JW and CDW: article writing with contributions from other authors; $\mathrm{XLZ}$ : revision and supervision.

1. Marie PJ. Osteoblast dysfunctions in bone diseases: from cellular and molecula mechanisms to therapeutic strategies. Cell Mol Life Sci 2015; 72: 1347-1361.

2. Simmons CA, Matlis S, Thornton AJ, Chen S, Wang CY, Mooney DJ. Cyclic strain enhances matrix mineralization by adult human mesenchymal stem cells via the extracellular signalregulated kinase (ERK1/2) signaling pathway. J Biomech 2003: 36: 1087-1096.

3. Li Y, Ge C, Long JP, Begun DL, Rodriguez JA, Goldstein SA et al. Biomechanical stimulation of osteoblast gene expression requires phosphorylation of the RUNX2 transcription factor. J Bone Miner Res 2012; 27: 1263-1274.

4. Delaine-Smith RM, Reilly GC. Mesenchymal stem cell responses to mechanical stimuli. Muscles Ligaments Tendons J 2012; 2: 169-180. 
5. Kurpinski K, Chu J, Hashi C, Li S. Anisotropic mechanosensing by mesenchymal stem cells. Proc Natl Acad Sci USA 2006; 103: 16095-16100.

6. Hao J, Zhang Y, Jing D, Shen Y, Tang G, Huang S et al. Mechanobiology of mesenchymal stem cells: perspective into mechanical induction of MSC fate. Acta Biomater 2015; 20: 1-9.

7. Kelly DJ, Jacobs CR. The role of mechanical signals in regulating chondrogenesis and osteogenesis of mesenchymal stem cells. Birth Defects Res C Embryo Today 2010; 90 : 75-85.

8. Angele P, Yoo JU, Smith C, Mansour J, Jepsen KJ, Nerlich M et al. Cyclic hydrostatic pressure enhances the chondrogenic phenotype of human mesenchymal progenitor cells differentiated in vitro. J Orthop Res 2003; 21: 451-457.

9. Chen JC, Jacobs CR. Mechanically induced osteogenic lineage commitment of stem cells. Stem Cell Res Ther 2013; 4: 107.

10. Rittweger J, Frost HM, Schiess $H$, Ohshima H, Alkner B, Tesch P et al. Muscle atrophy and bone loss after 90 days' bed rest and the effects of flywheel resistive exercise and pamidronate: results from the LTBR study. Bone 2005; 36: 1019-1029.

11. Zwart SR, Pierson D, Mehta S, Gonda S, Smith SM. Capacity of omega-3 fatty acids or eicosapentaenoic acid to counteract weightlessness-induced bone loss by inhibiting NF-kappaB activation: from cells to bed rest to astronauts. J Bone Miner Res 2010; 25: 1049-1057.

12. Chen L, Holmstrom K, Qu W, Ditzel N, Shi K, Hokland L et al. MicroRNA-34a inhibits osteoblast differentiation and in vivo bone formation of human stromal stem cells. Stem Cells 2014; 32: 902-912.

13. Zuo B, Zhu JF, Li J, Wang CD, Zhao XY, Cai GQ et al. microRNA-103a functions as a mechno-sensitive microRNA to inhibit bone formation through targeting Runx2. J Bone Miner Res 2014; 30: 330-345.

14. Papaioannou G, Mirzamohammadi F, Kobayashi T. MicroRNAs involved in bone formation Cell Mol Life Sci 2014; 71: 4747-4761.

15. Hemming S, Cakouros D, Isenmann S, Cooper L, Menicanin D, Zannettino A et al. EZH2 and KDM6A act as an epigenetic switch to regulate mesenchymal stem cell lineage specification. Stem Cells 2014; 32: 802-815.

16. Huang $B$, Li G, Jiang XH. Fate determination in mesenchymal stem cells: a perspective from histone-modifying enzymes. Stem Cell Res Ther 2015; 6: 35

17. Reichert N, Choukrallah MA, Matthias P. Multiple roles of class I HDACs in proliferation, differentiation, and development. Cell Mol Life Sci 2012; 69: 2173-2187.

18. Haberland M, Montgomery RL, Olson EN. The many roles of histone deacetylases in development and physiology: implications for disease and therapy. Nat Rev Genet 2009; 10 : $32-42$.

19. Lee HW, Suh JH, Kim AY, Lee YS, Park SY, Kim JB. Histone deacetylase 1-mediated histone modification regulates osteoblast differentiation. Mol Endocrinol 2006; 20 : 2432-2443.

20. Maroni P, Brini AT, Arrigoni E, de Girolamo L, Niada S, Matteucci E et al. Chemical and genetic blockade of HDACs enhances osteogenic differentiation of human adipose tissuederived stem cells by oppositely affecting osteogenic and adipogenic transcription factors. Biochem Biophys Res Commun 2012; 428: 271-277.

21. Zhu F, Sweetwyne MT, Hankenson KD. PKCdelta is required for Jagged-1 induction of human mesenchymal stem cell osteogenic differentiation. Stem Cells 2013; 31: 1181-1192.

22. Shimizu T, Tanaka T, Iso T, Doi H, Sato H, Kawai-Kowase $\mathrm{K}$ et al. Notch signaling induces osteogenic differentiation and mineralization of vascular smooth muscle cells: role of Msx2 gene induction via Notch-RBP-Jk signaling. Arterioscler Thromb Vasc Biol 2009; 29 1104-1111.

23. Kung AW, Xiao SM, Cherny S, Li GH, Gao Y, Tso G et al. Association of JAG1 with bone mineral density and osteoporotic fractures: a genome-wide association study and follow-up replication studies. Am J Hum Genet 2010; 86: 229-239.

24. Benisch P, Schilling T, Klein-Hitpass L, Frey SP, Seefried L, Raaijmakers $N$ et al. The transcriptional profile of mesenchymal stem cell populations in primary osteoporosis is distinct and shows overexpression of osteogenic inhibitors. PLoS One 2012; 7: e45142.

25. Steward AJ, Kelly DJ. Mechanical regulation of mesenchymal stem cell differentiation. J Anat 2014; 227: 717-731.

26. Hoey DA, Tormey S, Ramcharan S, O'Brien FJ, Jacobs CR. Primary cilia-mediated mechanotransduction in human mesenchymal stem cells. Stem Cells 2012; 30: 2561-2570.

27. Sen B, Guilluy C, Xie Z, Case N, Styner M, Thomas J et al. Mechanically induced focal adhesion assembly amplifies anti-adipogenic pathways in mesenchymal stem cells. Stem Cells 2011; 29: 1829-1836.
28. Benham-Pyle BW, Pruitt BL, Nelson WJ. Cell adhesion. Mechanical strain induces E-cadherin-dependent Yap1 and beta-catenin activation to drive cell cycle entry. Science 2015; 348: 1024-1027.

29. Tezuka K, Yasuda M, Watanabe N, Morimura N, Kuroda K, Miyatani S et al. Stimulation of osteoblastic cell differentiation by Notch. J Bone Miner Res 2002; 17: 231-239.

30. Dishowitz Ml, Terkhorn SP, Bostic SA, Hankenson KD. Notch signaling components are upregulated during both endochondral and intramembranous bone regeneration. J Orthop Res 2012; 30: 296-303.

31. Oda T, Elkahloun AG, Pike BL, Okajima K, Krantz ID, Genin A et al. Mutations in the human Jagged1 gene are responsible for Alagille syndrome. Nat Genet 1997; 16: 235-242.

32. Ye L, Fan Z, Yu B, Chang J, Al Hezaimi K, Zhou X et al. Histone demethylases KDM4B and KDM6B promotes osteogenic differentiation of human MSCs. Cell Stem Cell 2012; 11: 50-61.

33. Paino F, La Noce M, Tirino V, Naddeo P, Desiderio V, Pirozzi G et al. Histone deacetylase inhibition with valproic acid downregulates osteocalcin gene expression in human dental pulp stem cells and osteoblasts: evidence for HDAC2 involvement. Stem Cells 2014; 32 : 279-289.

34. Chen LJ, Wei SY, Chiu JJ. Mechanical regulation of epigenetics in vascular biology and pathobiology. J Cell Mol Med 2013; 17: 437-448.

35. Lee DY, Lee Cl, Lin TE, Lim SH, Zhou J, Tseng YC et al. Role of histone deacetylases in transcription factor regulation and cell cycle modulation in endothelial cells in response to disturbed flow. Proc Natl Acad Sci USA 2012; 109: 1967-1972.

36. Zeng L, Zhang Y, Chien S, Liu X, Shyy JY. The role of p53 deacetylation in p21Waf1 regulation by laminar flow. J Biol Chem 2003; 278: 24594-24599.

37. Lee S, Park JR, Seo MS, Roh KH, Park SB, Hwang JW et al. Histone deacetylase inhibitors decrease proliferation potential and multilineage differentiation capability of human mesenchymal stem cells. Cell Prolif 2009; 42: 711-720.

38. Arnsdorf EJ, Tummala P, Castillo AB, Zhang F, Jacobs $C R$. The epigenetic mechanism of mechanically induced osteogenic differentiation. J Biomech 2010; 43: 2881-2886.

39. Song G, Ju Y, Shen X, Luo Q, Shi Y, Qin J. Mechanical stretch promotes proliferation of rat bone marrow mesenchymal stem cells. Colloids Surf B Biointerfaces 2007; 58: 271-277.

40. Luu YK, Capilla E, Rosen CJ, Gilsanz V, Pessin JE, Judex S et al. Mechanical stimulation of mesenchymal stem cell proliferation and differentiation promotes osteogenesis while preventing dietary-induced obesity. J Bone Miner Res 2009; 24: 50-61.

41. Terraciano V, Hwang N, Moroni L, Park HB, Zhang Z, Mizrahi J et al. Differential response of adult and embryonic mesenchymal progenitor cells to mechanical compression in hydrogels. Stem Cells 2007; 25: 2730-2738.

42. Hamilton DW, Maul TM, Vorp DA. Characterization of the response of bone marrow-derived progenitor cells to cyclic strain: implications for vascular tissue-engineering applications. Tissue Eng 2004; 10: 361-369.

43. Zhao F, Chella R, Ma T. Effects of shear stress on 3-D human mesenchymal stem cell construct development in a perfusion bioreactor system: experiments and hydrodynamic modeling. Biotechnol Bioeng 2007; 96: 584-595.

44. Isenmann S, Arthur A, Zannettino AC, Turner JL, Shi S, Glackin CA et al. TWIST family of basic helix-loop-helix transcription factors mediate human mesenchymal stem cell growth and commitment. Stem Cells 2009; 27: 2457-2468.

45. Subramanian A, Tamayo P, Mootha VK, Mukherjee S, Ebert BL, Gillette MA et al. Gene set enrichment analysis: a knowledge-based approach for interpreting genome-wide expression profiles. Proc Natl Acad Sci USA 2005; 102: 15545-15550.

Cell Death and Disease is an open-access journal published by Nature Publishing Group. This work is licensed under a Creative Commons Attribution 4.0 International License. The images or other third party material in this article are included in the article's Creative Commons license, unless indicated otherwise in the credit line; if the material is not included under the Creative Commons license, users will need to obtain permission from the license holder to reproduce the material. To view a copy of this license, visit http://creativecommons.org/licenses/by/4.0/ 